\title{
Manin's conjecture on a nonsingular quartic del Pezzo surface
}

by

FoK-Shuen Leung (Waterloo, ON)

1. Introduction. Let $V$ be the nonsingular del Pezzo surface of degree four defined by the zero locus of the equations

$$
\begin{aligned}
& 0=x_{1} x_{2}-x_{3} x_{4}, \\
& 0=x_{1}^{2}+x_{2}^{2}+x_{3}^{2}-x_{4}^{2}-2 x_{5}^{2} .
\end{aligned}
$$

Let $U \subseteq V$ be formed by deleting the lines from $V$. Given a rational point $\mathbf{x}=\left[x_{1}, \ldots, x_{5}\right] \in \mathbb{P}^{4}(\mathbb{Q})$ with $x_{1}, \ldots, x_{5} \in \mathbb{Z}$ and $\operatorname{gcd}\left(x_{1}, \ldots, x_{5}\right)=1$, we define the height of $\mathbf{x}$ to be $\|\mathbf{x}\|=\max \left(\left|x_{1}\right|, \ldots,\left|x_{5}\right|\right)$. Given $B \geq 1$, the density of rational points on $U$ is specified by the cardinality

$$
N_{U}(B)=\#\left\{\mathbf{x} \in U \cap \mathbb{P}^{4}(\mathbb{Q}):\|\mathbf{x}\| \leq B\right\} .
$$

Manin's conjecture, proposed in [4] for Fano varieties in general, predicts that if the set of rational points on $V$ is nonempty, then

$$
N_{U}(B)=c_{V} B(\log B)^{\varrho-1}(1+o(1))
$$

as $B \rightarrow \infty$, where $c_{V}$ is a positive constant and $\varrho$ is the rank of the Picard group of $V$. Our principal result is the following:

Theorem 1.1. $B(\log B)^{\varrho-1} \ll N_{U}(B) \ll B(\log B)^{\varrho-1}$.

An overview of progress in proving Manin's conjecture for del Pezzo surfaces can be found in [2]. In general, singular del Pezzo surfaces of low degree have proven more tractable than their nonsingular counterparts.

For nonsingular quartic del Pezzo surfaces, the best result until now is due to Salberger, who proved $N_{U}(B) \ll B^{1+\varepsilon}$ for any $\varepsilon>0$, provided $V$ contains a rational conic; this work was presented at the 2001 Budapest conference Higher dimensional varieties and rational points. Our result refines Salberger's. 
Both bounds comprising Theorem 1.1 involve fibering $V$ into a family of conics; this allows us to reduce the problem of estimating $N_{U}(B)$ to the problem of estimating the density of certain rational points on these conics. The same idea is central to Salberger's result; our improved bound stems from tighter control on the uniformity of bounds for rational points on the conics. The method appears to be applicable in a far more general setting, and we intend to explore this in a future paper.

2. The constant $\varrho$. We begin by recounting some geometry of quartic del Pezzo surfaces. We refer the reader to [5] for a comprehensive exposition.

In general, a nonsingular quartic del Pezzo surface $X$ contains 16 lines, each of which intersects exactly five others. Given any subset of five pairwise skew lines $L_{1}, \ldots, L_{5}, X$ is isomorphic to $\mathbb{P}^{2}$ blown up along five points $P_{1}, \ldots, P_{5}$ in general position such that $L_{1}, \ldots, L_{5}$ are the preimages of those points under the blowup. Moreover, there exists a unique line $L_{0}$ intersecting $L_{1}, \ldots, L_{5} ; L_{0}$ is the preimage of the unique conic on $\mathbb{P}^{2}$ through $P_{1}, \ldots, P_{5}$.

Let $K_{0}, \ldots, K_{6}$ denote the linear equivalence classes of $L_{0}, \ldots, L_{6}$, respectively, and $K$ denote the class of the preimage of a line on $\mathbb{P}^{2}$. Then

$$
K_{0} \sim 2 K-\left(K_{1}+\cdots+K_{5}\right) .
$$

The geometric Picard group of $X$ - that is, the Picard group of $X$ defined over an extension $E$ of minimal degree over $\mathbb{Q}$ such that all the lines on $X$ are defined over $E$ - has a basis $\left\{K, K_{1}, \ldots, K_{5}\right\}$. The Picard group of $X$ is that part of the geometric Picard group invariant under the action of $\operatorname{Gal}(E / \mathbb{Q})$.

The 16 lines on $V$ have the following parametrizations:

$$
\begin{array}{ll}
L_{1}:[a, b, a, b, a], & L_{2}:[a, b, a, b,-a], \\
L_{3}:[a, b,-a,-b, a], & L_{4}:[a, b,-a,-b,-a], \\
L_{5}:[a, b, b, a, b], & L_{6}:[a, b, b, a,-b], \\
L_{7}:[a, b,-b,-a, b], & L_{8}:[a, b,-b,-a,-b], \\
L_{9}:[a, b, i a,-i b, b], & L_{10}:[a, b, i a,-i b,-b], \\
L_{11}:[a, b,-i a, i b, b], & L_{12}:[a, b,-i a, i b,-b], \\
L_{13}:[a, b,-i b, i a, a], & L_{14}:[a, b,-i b, i a,-a], \\
L_{15}:[a, b, i b,-i a, a], & L_{16}:[a, b, i b,-i a,-a] .
\end{array}
$$

Note that all the lines are defined over $\mathbb{Q}(i)$. Let $K_{0}, \ldots, K_{5}$ denote the classes of $L_{5}, L_{1}, L_{4}, L_{6}, L_{9}$ and $L_{11}$, respectively. Note that the latter five lines are pairwise skew, and that they are intersected by $L_{5}$. Let $K$ denote 
the class of the preimage of a line on $\mathbb{P}^{2}$. In view of $(2.1)$, since $K_{0}, K_{1}$, $K_{2}, K_{3}$ and $K_{4}+K_{5}$ are invariant under the action of $\operatorname{Gal}(\mathbb{Q}(i) / \mathbb{Q})$, so too is $K$; and since $\left\{K, K_{1}, \ldots, K_{5}\right\}$, being a basis, is a linearly independent set, the set $\left\{K, K_{1}, K_{2}, K_{3}, K_{4}+K_{5}\right\}$ is also linearly independent. Therefore the Picard group of $V$ has rank at least 5. Since not all the lines on $V$ are invariant under the action of $\operatorname{Gal}(\mathbb{Q}(i) / \mathbb{Q})$, we conclude that the Picard group of $V$ has rank exactly 5 .

\section{The lower bound}

3.1. Preliminaries. Let $B>0$ be given and $P=\left\{(r, s): s\right.$ is even, $\operatorname{gcd}(r, s)=1$ and $\left.1 \leq r, s \leq B^{1 / 100}\right\}$.

Given $(r, s) \in P$, the first quadric of $V$ is satisfied by taking $x_{1}=r X_{1}$, $x_{2}=s X_{2}, x_{3}=s X_{1}$ and $x_{4}=r X_{2}$; and setting $x_{5}=X_{3}$, the second quadric of $V$ is a ternary quadric $0=Q_{r, s}(\mathbf{X})$, where

$$
Q_{r, s}(\mathbf{X})=\left(r^{2}+s^{2}\right) X_{1}^{2}-\left(r^{2}-s^{2}\right) X_{2}^{2}-2 X_{3}^{2} .
$$

If $\operatorname{gcd}\left(X_{1}, X_{2}, X_{3}\right)=1$, then $\|\mathbf{x}\| \leq B$ is implied by the bounds

$$
\left|X_{1}\right|,\left|X_{2}\right| \leq \frac{B}{\max (r, s)}, \quad\left|X_{3}\right| \leq B
$$

Let

$$
N_{r, s}=\#\left\{\mathbf{X}: 0=Q_{r, s}(\mathbf{X}), \operatorname{gcd}\left(X_{1}, X_{2}, X_{3}\right)=1 \text { and (3.1) holds }\right\},
$$

and let $P_{i}$ denote the set of pairs $(r, s) \in P$ in the dyadic ranges

$$
2^{i-1}=R_{i}<r \leq 2 R_{i}=2^{i}, \quad 2^{i}=S_{i}<s \leq 2 S_{i}=2^{i+1} .
$$

(Note that, given $(r, s) \in P_{i}$ for any $i$, we have $r<s$.) Then

$$
N_{U}(B) \gg \sum_{i} \sum_{(r, s) \in P_{i}} N_{r, s},
$$

where the $i$ are summed over those values such that the sets $P_{i}$ are nonempty.

3.2. The cardinality $N_{r, s}$. Let $(r, s) \in P_{i}$ be given. We estimate $N_{r, s}$ by parametrizing a subset of rational points on the quadric $0=Q_{r, s}(\mathbf{X})$.

We begin by observing that $[1,1, s]$ is a point on $0=Q_{r, s}(\mathbf{X})$. We fix a nonzero integer constant $c$, and consider all points on the quadric of the form

$$
\mathbf{X}=[c+x+2 s y, c+x, c s],
$$

where $(x, y)$ is an integer pair satisfying the coprimality condition

$$
\operatorname{gcd}(x, 2 s y)=1 \text {. }
$$


Note that distinct pairs $(x, y)$ parametrize distinct points $\mathbf{X}$. We proceed to eliminate the constant $c$. Substituting $\mathbf{X}$ back into $0=Q_{r, s}(\mathbf{X})$, we get

$$
0=\left(r^{2}+s^{2}\right)\left((x+2 s y)^{2}+2 c(x+2 s y)\right)-\left(r^{2}-s^{2}\right)\left(x^{2}+2 c x\right) .
$$

We rearrange this to get

$$
c f_{r, s}(x, y)=-\left(r^{2}+s^{2}\right)(x+2 s y)^{2}+\left(r^{2}-s^{2}\right) x^{2},
$$

where

$$
f_{r, s}(x, y)=2\left(r^{2}+s^{2}\right)(x+2 s y)-2 x\left(r^{2}-s^{2}\right) .
$$

We simplify $\mathbf{X}$ by multiplying each of its components by $f_{r, s}(x, y)$ and then dividing out by $s^{2}$, getting $\mathbf{X}=\left[f_{1, r, s}(x, y), f_{2, r, s}(x, y), f_{3, r, s}(x, y)\right]$, where

$$
\begin{aligned}
& f_{1, r, s}(x, y)=x^{2}+4 s x y+2\left(r^{2}+s^{2}\right) y^{2}, \\
& f_{2, r, s}(x, y)=x^{2}-2\left(r^{2}+s^{2}\right) y^{2}, \\
& f_{3, r, s}(x, y)=-s x^{2}-2\left(r^{2}+s^{2}\right) x y-2 s\left(r^{2}+s^{2}\right) y^{2} .
\end{aligned}
$$

Now given an integer pair $(x, y)$ satisfying (3.3), the forms $f_{1, r, s}(x, y)$, $f_{2, r, s}(x, y)$ and $f_{3, r, s}(x, y)$ may have a nontrivial common divisor:

Lemma 3.1. Let $(x, y)$ be an integer pair satisfying (3.3). Then the greatest common divisor of $f_{1, r, s}(x, y), f_{2, r, s}(x, y)$ and $f_{3, r, s}(x, y)$ is equal to

$$
\operatorname{gcd}\left(x, r^{2}+s^{2}\right) \operatorname{gcd}\left(x+2 s y, r^{2}-s^{2}\right) .
$$

Proof. Note that

$$
f_{1, r, s}(x, y)+f_{2, r, s}(x, y)=2 x(x+2 s y) .
$$

Now 2, $x$ and $x+2 s y$ are pairwise coprime; hence the greatest common divisor of $f_{1, r, s}(x, y), f_{2, r, s}(x, y)$ and $f_{3, r, s}(x, y)$ is equal to the product of the factors $\operatorname{gcd}\left(2, f_{2, r, s}(x, y), f_{3, r, s}(x, y)\right), \operatorname{gcd}\left(x, f_{2, r, s}(x, y), f_{3, r, s}(x, y)\right)$ and $\operatorname{gcd}\left(x+2 s y, f_{2, r, s}(x, y), f_{3, r, s}(x, y)\right)$. We denote these factors $F_{1}, F_{2}$ and $F_{3}$, respectively, and simplify each in turn. For the first, (3.3) implies that $x$, hence $f_{2, r, s}(x, y)$, is odd; thus $F_{1}=1$. For the second, we again apply (3.3), getting

$$
F_{2}=\operatorname{gcd}\left(x, 2\left(r^{2}+s^{2}\right) y^{2}, 2 s\left(r^{2}+s^{2}\right) y^{2}\right)=\operatorname{gcd}\left(x, r^{2}+s^{2}\right) .
$$

For the third, note that $f_{2, r, s}(x, y)=(x+2 s y)(x-2 s y)-2\left(r^{2}-s^{2}\right) y^{2}$ and $f_{3, r, s}(x, y)=-(x+2 s y)\left(s x+2 r^{2} y\right)+2 s\left(r^{2}-s^{2}\right) y^{2}$; hence

$$
F_{3}=\operatorname{gcd}\left(x+2 s y, 2\left(r^{2}-s^{2}\right) y^{2}, 2 s\left(r^{2}-s^{2}\right) y^{2}\right)=\operatorname{gcd}\left(x+2 s y, r^{2}-s^{2}\right),
$$

which completes the proof.

Suppose we have $\operatorname{gcd}\left(x, r^{2}+s^{2}\right) \operatorname{gcd}\left(x+2 s y, r^{2}-s^{2}\right)=n$. Then, given a point $\mathbf{X}=\left[f_{1, r, s}(x, y), f_{2, r, s}(x, y), f_{3, r, s}(x, y)\right]$, the bounds (3.1) are implied 
by the bounds

$$
\frac{\left|f_{1, r, s}(x, y)\right|}{n}, \frac{\left|f_{2, r, s}(x, y)\right|}{n} \leq \frac{B}{s}, \quad \frac{\left|f_{3, r, s}(x, y)\right|}{n} \leq B,
$$

which are themselves implied by the bounds

$$
1 \leq x \leq X=\left(\frac{B n}{4 s}\right)^{1 / 2}, \quad|y| \leq Y=\left(\frac{B n}{16 s^{3}}\right)^{1 / 2} .
$$

For convenience, we let $z=x+2 s y$, which allows us to replace the above bounds with $1 \leq x, z \leq X$.

We estimate $N_{r, s}$ by indexing the pairs $(x, z)$ contributing to $N_{r, s}$ according to the greatest common divisor of the components of $\mathbf{X}$. Let

$$
N_{n, r, s}=\#\left\{(x, z): \begin{array}{l}
\operatorname{gcd}(x, s z)=1,2 s \mid x-z, 1 \leq x, z \leq X, \\
\operatorname{gcd}\left(x, r^{2}+s^{2}\right) \operatorname{gcd}\left(z, r^{2}-s^{2}\right)=n
\end{array}\right\} .
$$

Then

$$
N_{r, s} \geq \sum_{n \geq 1} N_{n, r, s}
$$

The most cumbersome condition on $N_{n, r, s}$ is the last. In order to keep track of it, we redefine $N_{n, r, s}$ in terms of positive integer pairs $(a, b)$, where $\operatorname{gcd}\left(x, r^{2}+s^{2}\right)=a, \operatorname{gcd}\left(z, r^{2}-s^{2}\right)=b$ and $a b=n$. We write $x=a u$, $r^{2}+s^{2}=a c, z=b v$ and $r^{2}-s^{2}=b d$, where

$$
\operatorname{gcd}(u, c)=1 \quad \text { and } \operatorname{gcd}(v, d)=1 .
$$

The last condition on $N_{n, r, s}$ is implicit in these definitions. The coprimality condition $\operatorname{gcd}(x, s z)=1$ is implied by

$$
\operatorname{gcd}(a, v)=\operatorname{gcd}(u, b)=1=\operatorname{gcd}(u, v)=1=\operatorname{gcd}(u, s)=1 ;
$$

the divisibility condition $2 s \mid x-z$ is simply restated as

$$
2 s \mid a u-b v ;
$$

and the bounds $1 \leq x, z \leq X$ are implied by the bounds

$$
1 \leq u \leq U=\left(\frac{B b}{4 a s}\right)^{1 / 2}, \quad 1 \leq v \leq V=\left(\frac{B a}{4 b s}\right)^{1 / 2} .
$$

Thus, defining

$$
N_{a, b, r, s}=\#\{(u, v):(3.4)-(3.7) \text { hold }\},
$$

we have

$$
N_{r, s} \geq \sum_{a \mid r^{2}+s^{2}} \sum_{b \mid r^{2}-s^{2}} N_{a, b, r, s}
$$


3.3. The cardinality $N_{a, b, r, s}$. Let $(r, s) \in P_{i}, a \mid r^{2}+s^{2}$ and $b \mid r^{2}-s^{2}$ be given. We estimate $N_{a, b, r, s}$ by fixing $u$ and then estimating the number of $v$ such that $(u, v)$ contributes to $N_{a, b, r, s}$. Given $u$ such that $\operatorname{gcd}(u, s)=1$, let

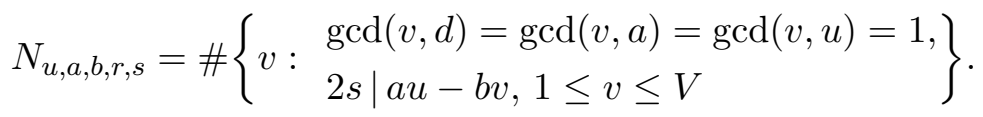

Then

$$
N_{a, b, r, s}=\sum_{u} N_{u, a, b, r, s}
$$

where the sum is taken over a suitable set of $u$. We shall define this set below.

We use the Möbius function to pick out the coprimality conditions on $N_{u, a, b, r, s}$. Let

$$
N_{u, a, b, r, s}^{\prime}\left(n_{1}, n_{2}, n_{3}\right)=\#\left\{v: \operatorname{lcm}\left(n_{1}, n_{2}, n_{3}\right)|v, 2 s| a u-b v, 1 \leq v \leq V\right\} .
$$

Then

$$
N_{u, a, b, r, s}=\sum_{n_{1} \mid d} \sum_{n_{2} \mid a} \sum_{n_{3} \mid u} \mu\left(n_{1}\right) \mu\left(n_{2}\right) \mu\left(n_{3}\right) N_{u, a, b, r, s}^{\prime}\left(n_{1}, n_{2}, n_{3}\right) .
$$

Let $n_{1}, n_{2}$ and $n_{3}$ be in the range of summation above. Then $\operatorname{gcd}\left(2 s, n_{1}\right)=1$, since $n_{1} \mid r^{2}-s^{2}$ and $\operatorname{gcd}\left(2 s, r^{2}-s^{2}\right)=1 ; \operatorname{gcd}\left(2 s, n_{2}\right)=1$, since $n_{2} \mid r^{2}+s^{2}$ and $\operatorname{gcd}\left(2 s, r^{2}+s^{2}\right)=1$; and $\operatorname{gcd}\left(2 s, n_{3}\right)=1$, since $\operatorname{gcd}(u, s)=1$ and $s$ is even. Moreover, $\operatorname{gcd}(2 s, b)=1$, since $b \mid r^{2}-s^{2}$. Thus

$$
N_{u, a, b, r, s}^{\prime}\left(n_{1}, n_{2}, n_{3}\right)=\frac{V}{2 s \cdot \operatorname{lcm}\left(n_{1}, n_{2}, n_{3}\right)}+O(1),
$$

and

$$
N_{u, a, b, r, s}=\sum_{n_{1} \mid d} \sum_{n_{2} \mid a} \sum_{n_{3} \mid u} \mu\left(n_{1}\right) \mu\left(n_{2}\right) \mu\left(n_{3}\right)\left(\frac{V}{2 s \cdot \operatorname{lcm}\left(n_{1}, n_{2}, n_{3}\right)}+O(1)\right) .
$$

We estimate $N_{a, b, r, s}$ by summing $N_{u, a, b, r, s}$ over the set

$$
P_{a, b, r, s}\left(n_{3}\right)=\left\{u: \operatorname{gcd}(u, c)=\operatorname{gcd}(u, b)=\operatorname{gcd}(u, s)=1, n_{3} \mid u, 1 \leq u \leq U\right\} ;
$$

that is, $N_{a, b, r, s}$ is equal to

$$
\sum_{n_{1} \mid d} \sum_{n_{2} \mid a} \sum_{n_{3} \leq U} \sum_{u \in P_{a, b, r, s}\left(n_{3}\right)} \mu\left(n_{1}\right) \mu\left(n_{2}\right) \mu\left(n_{3}\right)\left(\frac{V}{2 s \cdot \operatorname{lcm}\left(n_{1}, n_{2}, n_{3}\right)}+O(1)\right) .
$$

Since the cardinality of $P_{a, b, r, s}\left(n_{3}\right)$ has an upper bound $U / n_{3}$, the contribution to $N_{a, b, r, s}$ of the error term above is of order at most

$$
U \sum_{n_{1} \mid d} \sum_{n_{2} \mid a} \sum_{n_{3} \leq U} \frac{1}{n_{3}} \leq U\left(R_{i} S_{i} U\right)^{\varepsilon}
$$


for any $\varepsilon>0$, provided $i$ and $B$ are sufficiently large; that is,

$$
N_{a, b, r, s}=\frac{V}{2 s} \sum_{n_{1} \mid d} \sum_{n_{2} \mid a} \sum_{n_{3} \leq U} \sum_{u \in P_{a, b, r, s}\left(n_{3}\right)} \frac{\mu\left(n_{1}\right) \mu\left(n_{2}\right) \mu\left(n_{3}\right)}{\operatorname{lcm}\left(n_{1}, n_{2}, n_{3}\right)}+O\left(U\left(R_{i} S_{i} U\right)^{\varepsilon}\right) .
$$

We now estimate the cardinality of $P_{a, b, r, s}\left(n_{3}\right)$ more precisely. As in the case of $N_{u, a, b, r, s}$, we use the Möbius function to pick out coprimality conditions on the set. Let

$$
P_{a, b, r, s}^{\prime}\left(n_{3}, m_{1}, m_{2}, m_{3}\right)=\left\{u: \operatorname{lcm}\left(n_{3}, m_{1}, m_{2}, m_{3}\right) \mid u, 1 \leq u \leq U\right\} .
$$

Then

$$
\# P_{a, b, r, s}=\sum_{m_{1} \mid c} \sum_{m_{2} \mid b} \sum_{m_{3} \mid s} \mu\left(m_{1}\right) \mu\left(m_{2}\right) \mu\left(m_{3}\right) \# P_{a, b, r, s}^{\prime}\left(n_{3}, m_{1}, m_{2}, m_{3}\right) .
$$

Now

$$
\# P_{a, b, r, s}^{\prime}\left(n_{3}, m_{1}, m_{2}, m_{3}\right)=\frac{U}{\operatorname{lcm}\left(n_{3}, m_{1}, m_{2}, m_{3}\right)}+O(1) .
$$

The contribution to $N_{a, b, r, s}$ of the error term is of order at most

$$
\frac{V}{s} \sum_{n_{1} \mid d} \sum_{n_{2} \mid a} \sum_{n_{3} \leq U} \sum_{m_{1} \mid c} \sum_{m_{2} \mid b} \sum_{m_{3} \mid s} \frac{1}{\operatorname{lcm}\left(n_{1}, n_{2}, n_{3}\right)} \leq \frac{V}{s}\left(R_{i} S_{i} U\right)^{\varepsilon}
$$

for any $\varepsilon>0$, provided $i$ and $B$ are sufficiently large; that is, $N_{a, b, r, s}$ equals

$$
\begin{array}{r}
\frac{U V}{2 s} \sum_{n_{1} \mid d} \sum_{n_{2} \mid a} \sum_{n_{3} \leq U} \sum_{m_{1} \mid c} \sum_{m_{2} \mid b} \sum_{m_{3} \mid s} \frac{\mu\left(n_{1}\right) \mu\left(n_{2}\right) \mu\left(n_{3}\right) \mu\left(m_{1}\right) \mu\left(m_{2}\right) \mu\left(m_{3}\right)}{\operatorname{lcm}\left(n_{1}, n_{2}, n_{3}\right) \cdot \operatorname{lcm}\left(n_{3}, m_{1}, m_{2}, m_{3}\right)} \\
+O\left(\left(U+\frac{V}{s}\right)\left(R_{i} S_{i} U\right)^{\varepsilon}\right) .
\end{array}
$$

Finally, we estimate the main term above. Let $T_{a, b, r, s}$ denote this term, and $T_{a, b, r, s}^{\prime}$ denote $T_{a, b, r, s}$ but with the difference that, in $T_{a, b, r, s}^{\prime}$, we sum over all positive integers $n_{3}$ rather than over the range $n_{3} \leq U$. Now the difference between $T_{a, b, r, s}^{\prime}$ and $T_{a, b, r, s}$ is of order at most

$$
\frac{U V}{s} \sum_{n_{1} \mid d} \sum_{n_{2} \mid a} \sum_{n_{3}>U} \sum_{m_{1} \mid c} \sum_{m_{2} \mid b} \sum_{m_{3} \mid s} \frac{1}{n_{3}^{2}} \leq \frac{U V}{s}\left(R_{i} S_{i}\right)^{\varepsilon} \sum_{n_{3}>U} \frac{1}{n_{3}^{2}} \leq \frac{V}{s}\left(R_{i} S_{i}\right)^{\varepsilon}
$$

for any $\varepsilon>0$, provided $i$ and $B$ are sufficiently large; that is,

$$
N_{a, b, r, s}=T_{a, b, r, s}^{\prime}+O\left(\left(U+\frac{V}{s}\right)\left(R_{i} S_{i} U\right)^{\varepsilon}\right) .
$$

In order to estimate $T_{a, b, r, s}^{\prime}$, we define the condition

$$
n_{1}\left|d, \quad n_{2}\right| a, \quad m_{1}\left|c, \quad m_{2}\right| b \quad \text { and } \quad m_{3} \mid s,
$$


and the function $f_{a, b, r, s}\left(n_{1}, n_{2}, n_{3}, m_{1}, m_{2}, m_{3}\right)$ to be equal to

$$
\begin{cases}\frac{\mu\left(n_{1}\right) \mu\left(n_{2}\right) \mu\left(n_{3}\right) \mu\left(m_{1}\right) \mu\left(m_{2}\right) \mu\left(m_{3}\right)}{\operatorname{lcm}\left(n_{1}, n_{2}, n_{3}\right) \cdot \operatorname{lcm}\left(n_{3}, m_{1}, m_{2}, m_{3}\right)} & \text { if }(3.9) \text { holds, } \\ 0 & \text { otherwise. }\end{cases}
$$

Then

$$
T_{a, b, r, s}^{\prime}=\frac{U V}{2 s} \sum_{\substack{n_{i}, m_{i} \geq 1 \\ \text { for } 1 \leq i \leq 3}} f_{a, b, r, s}\left(n_{1}, n_{2}, n_{3}, m_{1}, m_{2}, m_{3}\right) .
$$

Because $f_{a, b, r, s}$ is multiplicative and we have

$$
\sum_{\substack{n_{i}, m_{i} \geq 1 \\ \text { for } 1 \leq i \leq 3}}\left|f_{a, b, r, s}\left(n_{1}, n_{2}, n_{3}, m_{1}, m_{2}, m_{3}\right)\right| \leq \sum_{n_{1} \mid d} \sum_{n_{2} \mid a} \sum_{m_{1} \mid c} \sum_{m_{2} \mid b} \sum_{m_{3} \mid 2 s} \sum_{n_{3} \geq 1} \frac{1}{n_{3}^{2}},
$$

which converges, we may write

$$
T_{a, b, r, s}^{\prime}=\frac{U V}{2 s} \prod_{p} f_{p, a, b, r, s},
$$

where the product is taken over all primes $p$, and the local factors $f_{p, a, b, r, s}$ are defined by

$$
f_{p, a, b, r, s}=\sum_{\substack{e_{i}, e_{i}^{\prime} \in\{0,1\} \\ \text { for } 1 \leq i \leq 3}} f_{a, b, r, s}\left(p^{e_{1}}, p^{e_{2}}, p^{e_{3}}, p^{e_{1}^{\prime}}, p^{e_{2}^{\prime}}, p^{e_{3}^{\prime}}\right) .
$$

We evaluate $f_{p, a, b, r, s}$ directly, in three cases. If $p$ does not divide any element in the set $\{a, b, c, d, s\}$, then $f_{p, a, b, r, s}=1-p^{-2}$; if $p$ divides exactly one element in the set $\{a, b, c, d, s\}$, then $f_{p, a, b, r, s}=1-p^{-1}$; and if $p$ divides exactly two elements in the set $\{a, b, c, d, s\}$-that is, either $p \mid a$ and $p \mid c$,or $p \mid b$ and $p \mid d$ - then $f_{p, a, b, r, s}=\left(1-p^{-1}\right)^{2}$. Hence

$$
\begin{aligned}
T_{a, b, r, s}^{\prime} & \geq \frac{U V}{2 s} \prod_{p \nmid s \Delta_{r, s}}\left(1-\frac{1}{p^{2}}\right) \prod_{p \mid s}\left(1-\frac{1}{p}\right) \prod_{p \mid \Delta_{r, s}}\left(1-\frac{1}{p}\right)^{2} \\
& \gg \frac{U V}{s} \prod_{p \mid s}\left(1-\frac{1}{p}\right) \prod_{p \mid \Delta_{r, s}}\left(1-\frac{1}{p}\right)^{2},
\end{aligned}
$$

where $\Delta_{r, s}$ denotes $\left|r^{4}-s^{4}\right|$, and the relation $\gg$ does not depend on our choice of $a, b, r$ or $s$. (For the remainder of this section we assume that all relations $\gg$ are thus independent.) Thus

$$
N_{a, b, r, s} \gg \frac{U V}{s} \prod_{p \mid s}\left(1-\frac{1}{p}\right) \prod_{p \mid \Delta_{r, s}}\left(1-\frac{1}{p}\right)^{2}+O\left(\left(U+\frac{V}{s}\right)\left(R_{i} S_{i} U\right)^{\varepsilon}\right)
$$


for any $\varepsilon>0$, provided $i$ and $B$ are sufficiently large. We conclude that

$$
N_{a, b, r, s} \gg \frac{B}{s^{2}} \prod_{p \mid s}\left(1-\frac{1}{p}\right) \prod_{p \mid \Delta_{r, s}}\left(1-\frac{1}{p}\right)^{2} .
$$

3.4. The cardinality $N_{U}(B)$. For convenience we define the multiplicative function

$$
f(n)=\prod_{p \mid n}\left(1-\frac{1}{p}\right)
$$

for any $n \in \mathbb{N}$, with $f(1)=1$. With this notation, and in view of the bounds (3.2), (3.8) and (3.10), we have

$$
\begin{aligned}
N_{U}(B) & \gg B \sum_{i} \frac{1}{S_{i}^{2}} \sum_{(r, s) \in P_{i}} \sum_{\substack{a\left|r^{2}+s^{2} \\
b\right| r^{2}-s^{2}}} f(s) f\left(\Delta_{r, s}\right)^{2} \\
& \geq B \sum_{i} \frac{1}{S_{i}^{2}} \sum_{(r, s) \in P_{i}} d\left(\Delta_{r, s}\right) f(s) f\left(\Delta_{r, s}\right)^{2},
\end{aligned}
$$

where we sum over those $i$ such that the $P_{i}$ are nonempty. We may restrict the range of summation on the right-hand side above without invalidating the bound, and it will be useful to impose the condition that, for any pair $(r, s)$ in that range of summation, $s$ is not only even but divisible by 6 ; that is,

$$
N_{U}(B) \gg B \sum_{i} \frac{1}{S_{i}^{2}} \sum_{\substack{S_{i}<s \leq 2 S_{i} \\ 6 \mid s}} f(s) \sum_{\substack{R_{i}<r \leq 2 R_{i} \\ \operatorname{gcd}(r, s)=1}} d\left(\Delta_{r, s}\right) f\left(\Delta_{r, s}\right)^{2} .
$$

We estimate the inner sum on the right-hand side of (3.11). Let $s$ be in the range of summation. By the Möbius inversion formula, we have

$$
d(n) f(n)^{2}=\sum_{m \mid n} f^{\prime}(m)
$$

for any $n \in \mathbb{N}$ if, and only if,

$$
f^{\prime}(n)=\sum_{m \mid n} \mu\left(\frac{n}{m}\right) d(m) f(m)^{2}
$$

for any $n \in \mathbb{N}$. Now $f^{\prime}$ is multiplicative, and given a prime power $p^{e}$ with $e \geq 1$, we have

$$
f^{\prime}\left(p^{e}\right)= \begin{cases}2(1-1 / p)^{2}-1 & \text { if } e=1, \\ (1-1 / p)^{2} & \text { otherwise }\end{cases}
$$

that is, $f^{\prime}\left(p^{e}\right)>0$ for any $e \in \mathbb{N}$ provided $p \geq 5$. No primes smaller than 5 
divide $\Delta_{r, s}$, since 2 and 3 both divide $s$; hence

$$
d\left(\Delta_{r, s}\right) f\left(\Delta_{r, s}\right)^{2}=\sum_{m \mid \Delta_{r, s}} f^{\prime}(m) \geq \sum_{\substack{m \mid \Delta_{r, s} \\ m \leq R_{i}^{1 / 2}}} f^{\prime}(m),
$$

where $f^{\prime}(m)$ is nonnegative over the range of summation. (It will shortly become clear why we impose a bound on $m$.) Thus

$$
\sum_{\substack{R_{i}<r \leq 2 R_{i} \\ \operatorname{gcd}(r, s)=1}} d\left(\Delta_{r, s}\right) f\left(\Delta_{r, s}\right)^{2} \geq \sum_{\substack{R_{i}<r \leq 2 R_{i} \\ \operatorname{gcd}(r, s)=1}} \sum_{\substack{m \mid \Delta_{r, s} \\ m \leq R_{i}^{1 / 2}}} f^{\prime}(m) .
$$

We use the Möbius function to pick out the coprimality condition on the right-hand side. As an intermediate step, we define

$$
N_{m, s}=\#\left\{r: R_{i}<r \leq 2 R_{i}, \operatorname{gcd}(r, s)=1, m \mid \Delta_{r, s}\right\} .
$$

Then

$$
\sum_{\substack{R_{i}<r \leq 2 R_{i} \\ \operatorname{gcd}(r, s)=1}} d\left(\Delta_{r, s}\right) f\left(\Delta_{r, s}\right)^{2} \geq \sum_{\substack{m \leq R_{i}^{1 / 2} \\ \operatorname{gcd}(m, s)=1}} f^{\prime}(m) N_{m, s}
$$

We impose the condition that $\operatorname{gcd}(m, s)=1$ on the range of summation on the right-hand side to ensure that the $N_{m, s}$ we sum are nonzero.

Let $N_{m, s}$ be nonzero; then the congruence $r^{4} \equiv s^{4}(\bmod m)$ is soluble in $r$, with $F(m)$ solutions $(\bmod m)$, where $F$ is a multiplicative function with

$$
F\left(2^{e}\right)=\left\{\begin{array}{ll}
2^{e-1} & \text { if } e \leq 4, \\
8 & \text { if } e>4,
\end{array} \quad F\left(p^{e}\right)= \begin{cases}2 & \text { if } p \equiv 3(\bmod 4) \\
4 & \text { if } p \equiv 1(\bmod 4)\end{cases}\right.
$$

Given a solution $r \equiv c(\bmod m)$, we define

$$
\begin{aligned}
N_{c, m, s} & =\#\left\{r: R_{i}<r \leq 2 R_{i}, \operatorname{gcd}(r, s)=1 \text { and } r \equiv c(\bmod m)\right\}, \\
N_{c, s}(n) & =\#\left\{r: R_{i}<r \leq 2 R_{i}, n \mid r \text { and } r \equiv c(\bmod m)\right\}
\end{aligned}
$$

then

$$
N_{c, m, s}=\sum_{n \mid s} \mu(n) N_{c, s}(n) .
$$

Let $n \mid s$. Then $\operatorname{gcd}(n, m)=1, \operatorname{since} \operatorname{gcd}(m, s)=1$. Thus

$$
N_{c, s}(n)=\frac{R_{i}}{n m}+O(1) .
$$

The contribution to $N_{c, m, s}$ of the error term above is of order at most $d(s)$; that is,

$$
N_{c, m, s}=\frac{R_{i}}{m} \sum_{n \mid s} \frac{\mu(n)}{n}+O(d(s))=\frac{R_{i} f(s)}{m}+O(d(s)) \gg \frac{R_{i} f(s)}{m} .
$$


(The above bound follows from the fact that $m \leq R_{i}^{1 / 2}$.) Thus

$$
N_{m, s} \gg \frac{F(m) R_{i} f(s)}{m},
$$

and

$$
\sum_{\substack{R_{i}<r \leq 2 R_{i} \\
\operatorname{gcd}(r, s)=1}} d\left(\Delta_{r, s}\right) f\left(\Delta_{r, s}\right)^{2} \gg R_{i} f(s) \sum_{\begin{array}{c}
m \leq R_{i}^{1 / 2} \\
\operatorname{gcd}(m, s)=1
\end{array}} \frac{F(m) f^{\prime}(m)}{m} .
$$

In view of the bound (3.11) and the fact that $R_{i}$ and $S_{i}$ are of the same order, we conclude that

$$
N_{U}(B) \gg B \sum_{i} \frac{1}{S_{i}} \sum_{\substack{m \leq R_{i}^{1 / 2} \\
\operatorname{gcd}(m, 6)=1}} \frac{F(m) f^{\prime}(m)}{m} \sum_{\begin{array}{c}
S_{i}<s \leq 2 S_{i} \\
6 \mid s \\
\operatorname{gcd}(m, s)=1
\end{array}} f(s)^{2},
$$

where we sum over those $i$ such that the sets $P_{i}$ are nonempty. (We impose the condition $\operatorname{gcd}(m, 6)=1$ for convenience.)

We proceed to estimate the inner sum on the right-hand side of (3.12). Let $s=6 t$ and $S_{i} / 6=T_{i}$. Then

$$
\sum_{\substack{S_{i}<s \leq 2 S_{i} \\ 6 \mid s \\ \operatorname{gcd}(m, s)=1}} f(s)^{2} \gg \sum_{\substack{T_{i}<t \leq 2 T_{i} \\ \operatorname{gcd}(m, t)=1}} f(t)^{2} .
$$

By the Cauchy-Schwarz inequality, we have

$$
\sum_{\substack{T_{i}<t \leq 2 T_{i} \\ \operatorname{gcd}(m, t)=1}} f(t)^{2} \geq\left(\sum_{\substack{T_{i}<t \leq 2 T_{i} \\ \operatorname{gcd}(m, t)=1}} 1\right)^{-1}\left(\sum_{\substack{T_{i}<t \leq 2 T_{i} \\ \operatorname{gcd}(m, t)=1}} f(t)\right)^{2} .
$$

We estimate the two sums on the right-hand side, using the following two standard relations: first, given any positive integer constant $c$, we have

$$
\#\{n: n \leq N \text { and } \operatorname{gcd}(n, c)=1\}=\frac{N \phi(c)}{c}+O\left(c^{\varepsilon}\right)
$$

for any $\varepsilon>0$; and second,

$$
\sum_{n \leq N} \phi(n)=\frac{3 N^{2}}{\pi^{2}}+O(N \log N) .
$$

For the first sum on the right-hand side of the Cauchy-Schwarz inequality, we have, by (3.13),

$$
\sum_{\substack{T_{i}<t \leq 2 T_{i} \\ \operatorname{gcd}(m, t)=1}} 1 \ll \frac{T_{i} \phi(m)}{m}=T_{i} f(m) .
$$


For the second sum, we have

$$
\sum_{\substack{T_{i}<t \leq 2 T_{i} \\ \operatorname{gcd}(m, t)=1}} f(t)=\sum_{\substack{T_{i}<t \leq 2 T_{i} \\ \operatorname{gcd}(m, t)=1}} \frac{\phi(t)}{t} \gg \frac{1}{T_{i}} \sum_{\substack{T_{i}<t \leq 2 T_{i} \\ \operatorname{gcd}(m, t)=1}} \phi(t) .
$$

By (3.13), we get

$$
\sum_{\substack{T_{i}<t \leq 2 T_{i} \\ \operatorname{gcd}(m, t)=1}} \phi(t) \geq \sum_{\substack{T_{i}<t \leq 2 T_{i} \\ \operatorname{gcd}(m s, t)=1}} \phi(t) \gg T_{i} \sum_{s \leq T_{i}} \frac{\phi(m s)}{m s} \geq T_{i} f(m) \sum_{s \leq T_{i}} f(s) ;
$$

that is,

$$
\sum_{\substack{T_{i}<t \leq 2 T_{i} \\ \operatorname{gcd}(m, t)=1}} f(t) \gg f(m) \sum_{s \leq T_{i}} f(s) \gg T_{i} f(m),
$$

where the second inequality follows from (3.14). Thus

$$
\sum_{\substack{T_{i}<t \leq 2 T_{i} \\ \operatorname{gcd}(m, t)=1}} f(t)^{2} \gg T_{i} f(m) \gg S_{i} f(m)
$$

and, in view of (3.12),

$$
N_{U}(B) \gg B \sum_{i} \sum_{\substack{m \leq R_{i}^{1 / 2} \\ \operatorname{gcd}(m, 6)=1}} \frac{F(m) f(m) f^{\prime}(m)}{m},
$$

where we sum over $i$ such that $P_{i} \neq \emptyset$.

We now estimate the inner sum on the right-hand side of (3.15). Since $F, f$ and $f^{\prime}$ are all multiplicative, we consider the corresponding Dirichlet series

$$
D(z)=\sum_{\substack{m \geq 1 \\ \operatorname{gcd}(m, 6)=1}} \frac{F(m) f(m) f^{\prime}(m)}{m^{z}}
$$

which admits an Euler product

$$
D(z)=\prod_{p \geq 5}\left(1+\frac{F(p) f(p) f^{\prime}(p)}{p^{z}}+\sum_{e \geq 2} \frac{F\left(p^{e}\right) f\left(p^{e}\right) f^{\prime}\left(p^{e}\right)}{p^{e z}}\right),
$$

where the product is taken over all primes $p \geq 5$. It is straightforward to rewrite this as $D(z)=\zeta(z)^{3} L(z, \chi) F^{\prime}(z)$, where $F^{\prime}$ is a holomorphic function bounded on the half-plane $\operatorname{Re}(z)>3 / 4$. Hence, by Perron's formula, the inner sum on the right-hand side of (3.15) is equal to

$$
\frac{1}{2 \pi i} \int_{\varepsilon-i T}^{\varepsilon+i T} \zeta(1+w)^{3} L(1+w, \chi) F^{\prime}(1+w) \frac{M^{w}}{w} d w+O(1) .
$$


The integrand has a pole of order 4 at $w=0$. We apply the residue theorem to the rectangular contour with corners at $\varepsilon-i T, \varepsilon+i T,-1 / 8+i T$ and $-1 / 8-i T$, and use the bounds

$$
\zeta(w), L(w, \chi) \ll|w|^{1 / 8},
$$

which hold provided $\operatorname{Re}(w) \geq 7 / 8$ and $|w-1| \geq 1 / 8$. These bounds imply that the integrand along the horizontal segments is of order at most

$$
\left(T^{1 / 8}\right)^{3} T^{1 / 8} \frac{M^{\operatorname{Re}(w)}}{T},
$$

where $-1 / 8 \leq \operatorname{Re}(w) \leq \varepsilon$; that is, the contribution of the integral along the horizontal segments of our contour is of order at most 1. Similarly, the integrand along the vertical segment joining $-1 / 8+i T$ to $-1 / 8-i T$ is of order at most

$$
\frac{\left(T^{1 / 8}\right)^{3} T^{1 / 8}}{M^{1 / 8}}
$$

that is, the contribution of the integral along that segment is of order at most

$$
\frac{T^{3 / 2}}{M^{1 / 8}}=M^{3 \varepsilon-1 / 8} \ll 1
$$

provided $\varepsilon<1 / 24$. Hence

$$
\sum_{\substack{m \leq M \\ \operatorname{gcd}(m, 6)=1}} \frac{F(m) f(m) f^{\prime}(m)}{m} \gg(\log M)^{3}=\left(\log R_{i}^{1 / 2}\right)^{3} .
$$

We insert the above bound into (3.15), getting

$$
N_{U}(B) \gg B \sum_{i}\left(\log R_{i}^{1 / 2}\right)^{3}
$$

where we sum over $i$ such that $P_{i} \neq \emptyset$. Now a set $P_{i}$ is nonempty provided $2^{i+1} \leq B^{1 / 100}$, that is, provided we have $i \leq k \log B$ for some fixed constant $k>0$. Thus

$$
N_{U}(B) \gg B \sum_{i \leq k \log B}\left(\log R_{i}^{1 / 2}\right)^{3} \gg B \sum_{i \leq k \log B}(i-1)^{3} \gg B(\log B)^{4} .
$$

\section{The upper bound}

4.1. Preliminaries. We define the following projections from $V$ onto $\mathbb{P}^{1}$ :

$$
\begin{aligned}
& f^{(1)}:\left[x_{1}, \ldots, x_{5}\right] \mapsto \begin{cases}{\left[x_{1}, x_{3}\right]} & \text { if }\left(x_{1}, x_{3}\right) \neq(0,0), \\
{\left[x_{4}, x_{2}\right]} & \text { otherwise, }\end{cases} \\
& f^{(2)}:\left[x_{1}, \ldots, x_{5}\right] \mapsto \begin{cases}{\left[x_{1}, x_{4}\right]} & \text { if }\left(x_{1}, x_{4}\right) \neq(0,0), \\
{\left[x_{3}, x_{2}\right]} & \text { otherwise. }\end{cases}
\end{aligned}
$$

We have the following lemma: 
Lemma 4.1. $\left\|f^{(1)}(\mathbf{x})\right\| \cdot\left\|f^{(2)}(\mathbf{x})\right\| \leq\|\mathbf{x}\|$ for all $\mathbf{x} \in V$.

Proof. Let $\operatorname{gcd}\left(x_{1}, x_{2}, x_{3}, x_{4}\right)=n$, and let $m_{i j}$ denote $\operatorname{gcd}\left(x_{i}, x_{j}\right) n^{-1}$ for $1 \leq i, j \leq 4$. Then

$$
\frac{x_{1}}{n}=m_{13} m_{14}, \quad \frac{x_{2}}{n}=m_{23} m_{24}, \quad \frac{x_{3}}{n}=m_{13} m_{23}, \quad \frac{x_{4}}{n}=m_{14} m_{24} .
$$

Now either $\left\|f^{(1)}(\mathbf{x})\right\|=\left\|\left[x_{1}, x_{3}\right]\right\|$ or $\left\|f^{(1)}(\mathbf{x})\right\|=\left\|\left[x_{4}, x_{2}\right]\right\|$; in both cases we get $\left\|f^{(1)}(\mathbf{x})\right\|=\left\|\left[m_{14}, m_{23}\right]\right\|$. Similarly, $\left\|f^{(2)}(\mathbf{x})\right\|=\left\|\left[m_{13}, m_{24}\right]\right\|$.

We define, for $i=1,2$,

$$
N_{U}^{(i)}(B)=\#\left\{\mathbf{x} \in U:\|\mathbf{x}\| \leq B \text { and }\left\|f^{(i)}(\mathbf{x})\right\| \leq B^{1 / 2}\right\} .
$$

By Lemma 4.1, we have

$$
N_{U}(B) \leq N_{U}^{(1)}(B)+N_{U}^{(2)}(B) .
$$

We will bound the $N_{U}^{(i)}(B)$. In fact, it suffices to bound $N_{U}^{(1)}(B)$; the bound for $N_{U}^{(2)}(B)$ follows by symmetry.

Suppose $\mathbf{x}$ contributes to $N_{U}^{(1)}(B)$; say $f^{(1)}(\mathbf{x})=[r, s]$ with $\operatorname{gcd}(r, s)=1$. Then $\mathbf{x}$ is of the form $\left[r X_{1}, s X_{2}, s X_{1}, r X_{2}, x_{5}\right]$, where $X_{1}=\operatorname{gcd}\left(x_{1}, x_{3}\right)$ and $X_{2}=\operatorname{gcd}\left(x_{2}, x_{4}\right)$; and, upon setting $x_{5}=X_{3}$, the second quadric of $V$ is a ternary quadric $0=Q_{r, s}^{(1)}(\mathbf{X})$, where

$$
Q_{r, s}^{(1)}(\mathbf{X})=\left(r^{2}+s^{2}\right) X_{1}^{2}-\left(r^{2}-s^{2}\right) X_{2}^{2}-2 X_{3}^{2} .
$$

(Note that the condition $\mathbf{x} \in U$ ensures that $r^{4}-s^{4} \neq 0$.) The condition $\|\mathbf{x}\| \leq B$ implies

$$
\left|X_{1}\right|,\left|X_{2}\right| \leq \frac{B}{\max (r, s)}, \quad\left|X_{3}\right| \leq B
$$

Thus, defining

$$
N_{r, s}=\#\left\{\mathbf{X}: \operatorname{gcd}\left(X_{1}, X_{2}, X_{3}\right)=1,0=Q_{r, s}^{(1)}(\mathbf{X}) \text { and (4.1) holds }\right\},
$$

we have

$$
N_{U}^{(1)}(B) \ll \sum_{\substack{\operatorname{gcd}(r, s)=1 \\ 1 \leq r, s \leq B^{1 / 2}}} N_{r, s} .
$$

We split the set of suitable pairs $(r, s)$ into dyadic ranges, letting $P_{i, j}$ denote the set of coprime pairs $(r, s)$ in the range

$$
2^{i-1}=R_{i}<r \leq 2 R_{i}=2^{i} \text { and } 2^{j-1}=S_{j}<s \leq 2 S_{j}=2^{j} .
$$

The bounds $1 \leq r, s \leq B^{1 / 2}$ imply that the indices $i$ and $j$ have an upper bound $i, j \leq 2 \log B$. Thus

$$
N_{U}^{(1)}(B) \ll \sum_{i \leq 2 \log B} \sum_{j \leq i} \sum_{(r, s) \in P_{i, j}} N_{r, s}+\sum_{j \leq 2 \log B} \sum_{i \leq j} \sum_{(r, s) \in P_{i, j}} N_{r, s} .
$$


We bound the first of the terms on the right-hand side; the second term is dealt with similarly.

4.2. Tools. Our first tool, used to estimate $N_{r, s}$, may be found in [3]:

Lemma 4.2. Let $f \in \mathbb{Z}[\mathbf{X}]$ be a ternary quadratic form. Let $M$ denote its matrix representation $M$, let $\Delta=|\operatorname{det} M| \neq 0$, and let $\Delta_{0}$ denote the highest common factor of the $2 \times 2$ minors of $M$. Let

$$
N=\#\left\{\mathbf{X}: \operatorname{gcd}\left(X_{1}, X_{2}, X_{3}\right)=1,0=f(\mathbf{x}) \text { and }\left|x_{i}\right| \leq B_{i} \text { for } i=1,2,3\right\} .
$$

Then

$$
N \ll\left(1+\left(\frac{B_{1} B_{2} B_{3} \Delta_{0}^{2}}{\Delta}\right)^{1 / 3}\right) d(\Delta) .
$$

We require some notation for our next result. Given $f \in \mathbb{Z}[x]$ with no fixed prime divisors, the multiplicative function $\varrho_{f}(m)$ denotes the number of solutions $n(\bmod m)$ of $f(n) \equiv 0(\bmod m)$. We collect below some useful results on this function. The first three are classical, and may be found in [6], for example. The last is attributed in [1] to unpublished work by Stephan Daniel; a proof is given in [1, Lemma 2].

Lemma 4.3. Let $f \in \mathbb{Z}[x]$ be of degree $g$, have no fixed prime divisors, and be such that $\operatorname{Disc}(f) \neq 0$. Then:

(a) $\varrho_{f}(p) \leq g$

(b) $\varrho_{f}\left(p^{e}\right) \leq g p^{e-1}$ for all $e \in \mathbb{N}$;

(c) $\varrho_{f}\left(p^{e}\right)=\varrho_{f}(p)$ for all $e \in \mathbb{N}$, provided $p \nmid \operatorname{Disc}(f)$;

(d) $\varrho_{f}\left(p^{e}\right) \leq 2 g^{3} p^{e(1-1 / g)}$ for all $e \in \mathbb{N}$.

We are now ready to prove the following:

Lemma 4.4. Let $f \in \mathbb{Z}[x]$ be of degree 4 , have no fixed prime divisors, and be such that $\operatorname{Disc}(f) \neq 0$. Let $\alpha, \beta \in(0,1)$ and $N_{1}, N_{2} \geq 2$ be such that $N_{2}^{\alpha} \leq N_{2}-N_{1} \leq N_{2}$ and $\|f\|^{\beta} \leq N_{2}$. Then the sum

$$
\sum_{N_{1}<n \leq N_{2}} d(|f(n)|)
$$

is of order at most

$$
\left(N_{2}-N_{1}\right) \prod_{p \leq N_{2}}\left(1-\frac{\varrho_{f}(p)}{p}\right) \exp \left(\sum_{p \leq N_{2}} \frac{d(p) \varrho_{f}(p)}{p}+c \sum_{p \mid \operatorname{Disc}(f)} \frac{1}{p}\right)
$$

for a constant $c>0$, where the implied constant depends only on $\alpha$ and $\beta$.

Proof. This is a special case of the main theorem in [7]. Nair's bound depends implicitly on the discriminant $\operatorname{Disc}(f)$. This dependence arises in two places in [7]. In both instances we may make it explicit or remove it. 
The first instance is in [7, Lemma 2], in the implied constant of the bound

$$
\sum_{n \leq N}\left(\frac{n}{\phi(n)}\right)^{4} \frac{d(n) \varrho_{f}(n)}{n} \ll \exp \left(\sum_{p \leq N}\left(\frac{p}{\phi(p)}\right)^{4} \frac{d(p) \varrho_{f}(p)}{p}\right) .
$$

We make this dependence explicit. We begin with the fact that

$$
\sum_{n \leq N}\left(\frac{n}{\phi(n)}\right)^{4} \frac{d(n) \varrho_{f}(n)}{n} \leq \exp \left(\sum_{p \leq N}\left(\frac{p}{\phi(p)}\right)^{4} \sum_{e \geq 1} \frac{d\left(p^{e}\right) \varrho_{f}\left(p^{e}\right)}{p^{e}}\right) .
$$

We shall make use of the bound

$$
\sum_{e \geq E} \frac{e+1}{n^{e}} \ll \frac{1}{n^{E}}\left(\frac{n}{n-1}\right)^{2},
$$

which holds for all $n \in \mathbb{N}$. (Here the relation « depends only on $E$.) Now given $p$ such that $p \nmid \operatorname{Disc}(f)$, by Lemma 4.3(a), Lemma 4.3(c) and (4.4), we have

$$
\sum_{e \geq 2} \frac{d\left(p^{e}\right) \varrho_{f}\left(p^{e}\right)}{p^{e}} \leq 4 \sum_{e \geq 2} \frac{e+1}{p^{e}} \ll \frac{1}{p^{2}} .
$$

Likewise, given $p$ such that $p \mid \operatorname{Disc}(f)$, by Lemma 4.3(d) and (4.4), we have

$$
\sum_{e \geq 8} \frac{d\left(p^{e}\right) \varrho_{f}\left(p^{e}\right)}{p^{e}} \leq 128 \sum_{e \geq 8} \frac{e+1}{p^{e / 4}} \ll \frac{1}{p^{2}} .
$$

Finally, given $p$ such that $p \mid \operatorname{Disc}(f)$, by Lemma 4.3(b), we have

$$
\sum_{2 \leq e<8} \frac{d\left(p^{e}\right) \varrho_{f}\left(p^{e}\right)}{p^{e}} \leq 4 \sum_{e<8} \frac{e+1}{p} \ll \frac{1}{p} .
$$

These bounds combine to give

$$
\sum_{n \leq N}\left(\frac{n}{\phi(n)}\right)^{4} \frac{d(n) \varrho_{f}(n)}{n} \ll \exp \left(\sum_{p \leq N}\left(\frac{p}{\phi(p)}\right)^{4} \frac{d(p) \varrho_{f}(p)}{p}+c \sum_{p \mid \operatorname{Disc}(f)} \frac{1}{p}\right)
$$

for a constant $c>0$, where the relation $\ll$ does not depend on $\operatorname{Disc}(f)$. The difference between this bound and (4.3) accounts for the difference between Lemma 4.4 and the main result in [7].

The second place in [7] in which a dependence on $\operatorname{Disc}(f)$ arises is in the author's reduction of the bound $[7,(6.3)]$, where, given a positive integer $n$ such that $N^{1 / 2}<n \leq N$, the bound $\varrho_{f}(n) \ll N^{1 / 8}$ is invoked; Disc $(f)$ figures in the implied constant. We remove the dependence on $\operatorname{Disc}(f)$ by invoking the bound $\varrho_{f}(n) \ll n^{4 / 5}$ for all $n \in \mathbb{N}$, where the relation $\ll$ does not depend on $\operatorname{Disc}(f)$; this proves to be sufficient.

We use Lemma 4.4 to prove our version of a result due to Browning and de la Bretèche, which we use to sum our estimates for $N_{r, s}$ over the pairs 
$(r, s) \in P_{i, j}$. We require a generalization of the function $\varrho_{f}$ to binary forms. Let $f \in \mathbb{Z}\left[x_{1}, x_{2}\right]$ have no fixed prime divisors. Then $\varrho_{f(1, x)}(m)$ denotes the number of solutions $n(\bmod m)$ of $f(1, n) \equiv 0(\bmod m)$, and we define for any prime $p$ the function

$$
\varrho_{f}^{*}(p)= \begin{cases}\varrho_{f(1, x)}(p)+1 & \text { if } p \mid f(0,1), \\ \varrho_{f(1, x)}(p) & \text { otherwise. }\end{cases}
$$

TheOREM 4.5. Let $f \in \mathbb{Z}\left[x_{1}, x_{2}\right]$ be of degree 4 , have no fixed prime divisors, and be such that $\operatorname{Disc}(f) \neq 0$ and $f(1,0) f(0,1) \neq 0$. Let $\alpha, \beta \in$ $(0,1)$ and $N, N_{1}, N_{2} \geq 2$ be such that $N_{2}^{\alpha} \leq N_{2}-N_{1} \leq N_{2}$ and $\min \left(N, N_{2}\right) \geq$ $a \max \left(N, N_{2}\right)^{4 \beta}\|f\|^{\beta}$ for a constant $a>0$ dependent only on $\beta$. Then

$$
\sum_{1 \leq n_{1} \leq N} \sum_{N_{1}<n_{2} \leq N_{2}} d\left(\left|f\left(n_{1}, n_{2}\right)\right|\right) \ll N\left(N_{2}-N_{1}\right) T,
$$

where

$$
T=\prod_{p \mid \operatorname{Disc}(f)}\left(1+\frac{1}{p}\right)^{b} \exp \left(c \sum_{p \mid \operatorname{Disc}(f)} \frac{1}{p}\right) \exp \left(\sum_{p \leq \max \left(N, N_{2}\right)} \frac{\varrho_{f}^{*}(p)}{p}\right)
$$

for constants $b, c>0$, and the relation $\ll$ depends only on $\alpha$ and $\beta$.

Proof. This theorem is an adaptation of [1, Theorem 1]. There, the authors take $n_{2} \leq N_{2}$; we take a shorter range of summation and appeal to Lemma 4.4. The condition that $f$ have no fixed prime divisors is required in the proof of the main theorem in [7], and implicit in the proof of Lemma 4.4. As in $[1, \S 3]$, we fix $n_{1}$ and consider the sum

$$
\sum_{N_{1}<n_{2} \leq N_{2}} d\left(\left|f\left(n_{2}\right)\right|\right) .
$$

By Lemma 4.4, the above sum has an upper bound of order at most

$$
\left(N_{2}-N_{1}\right) \prod_{p \leq N_{2}}\left(1-\frac{\varrho_{f}(p)}{p}\right) \exp \left(\sum_{p \leq N_{2}} \frac{d(p) \varrho_{f}(p)}{p}+c \sum_{p \mid n_{1} \operatorname{Disc}(f)} \frac{1}{p}\right)
$$

for a constant $c>0$. In comparison, in $[1, \S 3]$, the authors conclude that

$$
\sum_{n_{2} \leq N_{2}} d\left(\left|f\left(n_{2}\right)\right|\right) \ll N_{2} \prod_{p \leq N_{2}}\left(1-\frac{\varrho_{f}(p)}{p}\right) \sum_{n_{2} \leq N_{2}} \frac{d\left(n_{2}\right) \varrho_{f}\left(n_{2}\right)}{n_{2}} .
$$

This difference accounts for the discrepancy between Theorem 4.5 and [1, Theorem 1]. Proceeding according to the argument of $[1, \S 3]$, we have

$$
\sum_{1 \leq n_{1} \leq N} \sum_{N_{1}<n_{2} \leq N_{2}} d\left(\left|f\left(n_{1}, n_{2}\right)\right|\right) \ll N\left(N_{2}-N_{1}\right) t_{1} t_{2},
$$


where

$$
\begin{aligned}
& t_{1}=\prod_{4<p \leq N_{2}}\left(1-\frac{\varrho_{f(1, x)}(p)}{p}\right) \exp \left(\sum_{\substack{p \leq N_{2} \\
p \nmid n_{1}}} \frac{d(p) \varrho_{f}(p)}{p}\right), \\
& t_{2}=\prod_{p \mid \operatorname{Disc}(f)}\left(1+\frac{1}{p}\right)^{b} \exp \left(c \sum_{p \mid \operatorname{Disc}(f)} \frac{1}{p}\right)
\end{aligned}
$$

for constants $b, c>0$. It is straightforward to show that $t_{1}$ is of order at most

$$
\prod_{4<p \leq N_{2}}\left(1-\frac{\varrho_{f(1, x)}(p)}{p}\right) \exp \left(\sum_{4<p \leq N_{2}} \frac{2 \varrho_{f(1, x)}(p)}{p}\right) \ll \exp \left(\sum_{p \leq N_{2}} \frac{\varrho_{f(1, x)}(p)}{p}\right),
$$

which, combined with $t_{2}$, yields the theorem.

Our third main tool is a classical result due to Dedekind and Landau:

Lemma 4.6. Let $f \in \mathbb{Z}[x]$ be irreducible and of degree $g \geq 1$. Then

$$
\sum_{p \leq B} \varrho_{f}(p)=\operatorname{Li}(B)+\left(\frac{B}{\exp \left(c(\log B)^{1 / 2}\right)}\right)
$$

for a constant $c>0$ dependent only on the splitting field of $f$ over $\mathbb{Q}$.

Proof. Let $L$ be the splitting field of $f$ over $\mathbb{Q}$. For all but finitely many $p$, $f(\bmod p)$ has factorization $F_{1} \cdots F_{n}(\bmod p)$, where each $F_{i} \in \mathbb{Z}_{p}[x]$ is irreducible and of degree $g_{i}$, if and only if the principal ideal $(p)$ has factorization $P_{1} \cdots P_{n}$, where each $P_{i}$ is a prime ideal over $L$ with norm $p^{g_{i}}$. Now

$$
\varrho_{f}(p)=\#\left\{i: F_{i} \text { is linear }\right\}=\#\left\{i: \operatorname{norm}\left(P_{i}\right)=p\right\},
$$

and by Landau's Prime Ideal Theorem,

$$
\#\{\text { prime ideals } P: \operatorname{norm}(P)=p \leq B\}=\operatorname{Li}(B)+O\left(\frac{B}{\exp \left(c(\log B)^{1 / 2}\right)}\right)
$$

for a constant $c>0$ dependent only on $L$.

Now the Prime Ideal Theorem is simply the generalization to number fields of the Prime Number Theorem; given $n \in \mathbb{N}$, we have

$$
\pi(n)=\operatorname{Li}(n)+O\left(\frac{n}{\exp \left(c^{\prime}(\log n)^{1 / 2}\right)}\right)
$$

for a constant $c^{\prime}>0$. This symmetry between $\pi(t)$ and the average order of $\varrho_{f}(p)$ will be useful. We also record the following bound, due to Rosser and Schoenfeld [8]:

LEMma 4.7. Let $n \geq 67$. Then

$$
\frac{n}{\log n-1 / 2}<\pi(n)<\frac{n}{\log n-3 / 2} .
$$


4.3. The proof of the upper bound. As in $\S 3$, we let $\Delta_{r, s}$ denote $\left|r^{4}-s^{4}\right|$. We shall also write $P, R$ and $S$ for $P_{i, j}, R_{i}$ and $S_{j}$, respectively.

We begin by applying Lemma 4.2 to get

$$
\sum_{(r, s) \in P} N_{r, s} \ll B\left(\frac{1}{R^{2 / 3}} \sum_{(r, s) \in P} \frac{d\left(\Delta_{r, s}\right)}{\Delta_{r, s}^{1 / 3}}\right) .
$$

We evaluate the sum on the right-hand side according to the size of $\Delta_{r, s}$. Let the linear factors of $\Delta_{r, s}$ be denoted $\left|s-\alpha_{i} r\right|$ for $i=1,2,3,4$. We consider three cases:

Case I: $\quad R$ and $S$ are not of the same order;

Case II: $\quad R$ and $S$ are of the same order, and $\left|s-\alpha_{i} r\right|>R / 4$ for $i=1,2,3,4$; and

Case III: $R$ and $S$ are of the same order, and $\left|s-\alpha_{i} r\right| \leq R / 4$ for some $i \in\{1,2,3,4\}$. (We may assume moreover that $\alpha_{i}=1$ or -1 , for otherwise we have $\left|s-\alpha_{i} r\right|>R / 4$.)

Note that, since we are in search of an upper bound, we may apply selectively the coprimality condition on $P$.

In Case I, $\Delta_{r, s}$ is dominated by the $r^{4}$ term, and we have

$$
\sum_{(r, s) \in P} N_{r, s} \ll B\left(\frac{1}{R^{2}} \sum_{(r, s) \in P} d\left(\Delta_{r, s}\right)\right) .
$$

Now

$$
\sum_{(r, s) \in P} d\left(\Delta_{r, s}\right) \leq \sum_{s \leq 2} \sum_{\max \left(S, R^{1 / 2}\right)} d\left(\Delta_{r, s}\right) .
$$

We apply Theorem 4.5 to the right-hand side, getting

$$
\sum_{(r, s) \in P} d\left(\Delta_{r, s}\right) \ll \max \left(S, R^{1 / 2}\right) R T,
$$

where

$$
T=\prod_{p \mid \operatorname{Disc}\left(\Delta_{r, s}\right)}\left(1+\frac{1}{p}\right)^{b} \exp \left(c \sum_{p \mid \operatorname{Disc}\left(\Delta_{r, s}\right)} \frac{1}{p}\right) \exp \left(\sum_{p \leq 2 R} \frac{\varrho_{\Delta_{r, s}}^{*}(p)}{p}\right)
$$

for some constants $b, c>0$. The fact that $\operatorname{Disc}\left(\Delta_{r, s}\right)=128 i$ implies that the first two terms are $\ll 1$, whence

$$
T \ll \exp \left(\sum_{p \leq 2 R} \frac{\varrho_{\Delta_{r, s}}^{*}(p)}{p}\right) \ll \exp \left(\sum_{p \leq 2 R} \frac{\varrho_{\Delta_{1, x}}(p)}{p}\right) .
$$

We appeal to Lemmas 4.6 and 4.7. The sum on the far right-hand side is 
equal to

$$
\frac{1}{2 R} \sum_{p \leq 2 R} \varrho_{\Delta_{1, x}}(p)+\int_{1}^{2 R} \sum_{p \leq t} \varrho_{\Delta_{1, x}}(p) \frac{d t}{t^{2}}+O\left(\int_{1}^{2 R} \sum_{p \leq t} \varrho_{\Delta_{1, x}}(p) \frac{d t}{t^{3}}\right) .
$$

The first term is small. Indeed, let $f_{1}(x)=1+x^{2}, f_{2}(x)=1+x$ and $f_{3}(x)=1-x$. Then, by Lemma 4.6 , the first term is equal to

$$
\frac{1}{2 R}\left(\sum_{p \leq 2 R} \varrho_{f_{1}(x)}(p)+\sum_{p \leq 2 R} \varrho_{f_{2}(x)}(p)+\sum_{p \leq 2 R} \varrho_{f_{3}(x)}(p)\right)=O(1) .
$$

The error term is also small: by Lemma $4.3\left(\right.$ a) we have $\varrho_{1, x}(p) \leq 4$ for all primes $p$; that is,

$$
O\left(\int_{1}^{2 R} \sum_{p \leq t} \varrho_{\Delta_{1, x}}(p) \frac{d t}{t^{3}}\right)=O\left(\int_{1}^{2 R} \frac{d t}{t^{2}}\right)=O(1) .
$$

Thus

$$
T \ll \exp \left(\int_{67}^{2 R} \sum_{p \leq t} \varrho_{\Delta_{1, x}}(p) \frac{d t}{t^{2}}+O(1)\right) .
$$

By Lemma 4.7, we have

$$
\int_{67}^{2 R} \sum_{p \leq t} \varrho_{\Delta_{1, x}}(p) \frac{d t}{t^{2}}<\int_{67}^{2 R} \frac{1}{\pi(t)} \sum_{p \leq t} \varrho_{\Delta_{1, x}}(p) \frac{d t}{t(\log t-3 / 2)} .
$$

Lemma 4.6 gives

$$
\begin{aligned}
\sum_{p \leq t} \varrho_{\Delta_{1, x}}(p) & =\sum_{p \leq t} \varrho_{f_{1}(x)}(p)+\sum_{p \leq t} \varrho_{f_{2}(x)}(p)+\sum_{p \leq t} \varrho_{f_{3}(x)}(p) \\
& =3\left(\operatorname{Li}(t)+O\left(\frac{t}{\exp \left(c(\log t)^{1 / 2}\right)}\right)\right)
\end{aligned}
$$

for a constant $c>0$. We also have

$$
\pi(t)=\operatorname{Li}(t)+O\left(\frac{t}{\exp \left(c^{\prime}(\log t)^{1 / 2}\right)}\right)
$$

for a constant $c^{\prime}>0$. Let $C=\min \left(c, c^{\prime}\right)$. Then

$$
\frac{1}{\pi(t)} \sum_{p \leq t} \varrho_{x, 1}(p)=3+O\left(\frac{1}{\log t-3 / 2}\right) .
$$

Substituting back into (4.6), we get

$$
\begin{aligned}
\int_{67}^{2 R} \sum_{p \leq t} \varrho_{\Delta_{1, x}}(p) \frac{d t}{t^{2}} & <\int_{67}^{2 R} \frac{3 d t}{t(\log t-3 / 2)}+O\left(\int_{67}^{2 R} \frac{d t}{t(\log t-3 / 2)^{2}}\right) \\
& =\log (\log (2 R)-2 / 3)^{3}+O(1)<\log (\log B)^{3}+O(1) .
\end{aligned}
$$


Thus $T \ll(\log B)^{3}$, and

$$
\sum_{(r, s) \in P} d\left(\Delta_{r, s}\right) \ll \max \left(S, R^{1 / 2}\right) R(\log B)^{3} ;
$$

that is,

$$
\sum_{(r, s) \in P} N_{r, s} \ll \max \left(\frac{S}{R}, \frac{1}{R^{1 / 2}}\right) B(\log B)^{3}
$$

for Case I.

Case II is handled identically: as in Case I, we have $\Delta_{r, s} \gg R^{4}$, and the same bound (4.7) results.

In Case III, suppose $\alpha_{1} \in \mathbb{R}$ and $\left|s-\alpha_{1} r\right| \leq R / 4$. Then the bounds

$$
r\left|\alpha_{1}-\alpha_{i}\right|-\left|s-\alpha_{1} r\right| \leq\left|s-\alpha_{i} r\right| \leq r\left|\alpha_{1}-\alpha_{i}\right|+\left|s-\alpha_{1} r\right|
$$

for $i=2,3,4$ imply that $\Delta_{r, s}$ is of order $\left|s-\alpha_{1} r\right| R^{3}$. We split the set of values for $\left|s-\alpha_{1} r\right|$ into dyadic ranges

$$
2^{i-1}=B_{i}<\left|s-\alpha_{1} r\right| \leq 2 B_{i}=2^{i},
$$

where the index $i$ has an upper bound

$$
I=\left\lceil\frac{\log (R / 4)}{\log 2}\right\rceil=\frac{\log R}{\log 2}+O(1) .
$$

In view of (4.5), we have

$$
\sum_{(r, s) \in P} N_{r, s} \ll B\left(\frac{1}{R^{5 / 3}} \sum_{i \leq I} \frac{1}{B_{i}^{1 / 3}} \sum_{\substack{(r, s) \in P \\ B_{i}<\left|s-\alpha_{1} r\right| \leq 2 B_{i}}} d\left(\Delta_{r, s}\right)\right) .
$$

Now

$$
\sum_{\substack{(r, s) \in P \\ B_{i}<\left|s-\alpha_{1} r\right| \leq 2 B_{i}}} d\left(\Delta_{r, s}\right) \leq \sum_{1 \leq s \leq 2 S} \sum_{K_{i} \leq r \leq L_{i}} d\left(\Delta_{r, s}\right)
$$

where

$$
K_{i}=\max \left(1, s-2 \max \left(B_{i}, S^{1 / 3}\right)\right), \quad L_{i}=\min \left(2 R, s+2 \max \left(B_{i}, S^{1 / 3}\right)\right) .
$$

We apply Theorem 4.5 to the right-hand side of (4.8), getting

$$
\sum_{\substack{(r, s) \in P \\ B_{i}<\left|s-\alpha_{1} r\right| \leq 2 B_{i}}} d\left(\Delta_{r, s}\right) \ll \max \left(B_{i}, S^{1 / 3}\right) S(\log B)^{3},
$$

hence

$$
\sum_{(r, s) \in P} N_{r, s} \ll\left(\frac{S}{R^{5 / 3}} \sum_{i \leq I} \frac{\max \left(B_{i}, S^{1 / 3}\right)}{B_{i}^{1 / 3}}\right) B(\log B)^{3} .
$$


If $\max \left(B_{i}, S^{1 / 3}\right)=B_{i}$, then

$$
\sum_{i \leq I} \frac{\max \left(B_{i}, S^{1 / 3}\right)}{B_{i}^{1 / 3}} \ll 2^{2 I / 3}=\exp \left(\frac{2 I}{3} \log 2\right) \ll R^{2 / 3},
$$

and if $\max \left(B_{i}, S^{1 / 3}\right)=S^{1 / 3}$, then

$$
\sum_{i \ll \log R} \frac{\max \left(B_{i}, S^{1 / 3}\right)}{B_{i}^{1 / 3}} \ll S^{1 / 3} .
$$

Thus we have, for Case III, the bound

$$
\sum_{(r, s) \in P} N_{r, s} \ll \max \left(\frac{S}{R}, \frac{S^{4 / 3}}{R^{5 / 3}}\right) B(\log B)^{3}=\frac{S}{R} B(\log B)^{3} .
$$

Comparing the bounds (4.7) and (4.9), we conclude that

$$
\sum_{(r, s) \in P} N_{r, s} \ll \max \left(\frac{S}{R}, \frac{1}{R^{1 / 2}}\right) B(\log B)^{3} .
$$

5. The cardinality $N_{U}(B)$. By the bounds (4.2) and (4.10), we have

$$
\sum_{i \leq 2 \log B} \sum_{j \leq i} \sum_{(r, s) \in P_{i, j}} N_{r, s} \ll B(\log B)^{3} \sum_{i \leq 2 \log B} \sum_{j \leq i} \max \left(\frac{S_{j}}{R_{i}}, \frac{1}{R_{i}^{1 / 2}}\right) .
$$

If $S_{j} \geq R_{i}^{1 / 2}$, the sum on the right-hand side is equal to

$$
\sum_{i \leq 2 \log B} \sum_{j \leq i} \frac{1}{2^{i-j}} \leq \sum_{i \leq 2 \log B} \sum_{j \geq 0} \frac{1}{2^{j}} \ll \log B
$$

otherwise, it is equal to

$$
\sum_{i \leq 2 \log B} \sum_{j \leq i} \frac{1}{2^{(i-1) / 2}} \ll 1
$$

Thus we have

$$
\sum_{i \leq 2 \log B} \sum_{j \leq i} \sum_{(r, s) \in P_{i, j}} N_{r, s} \ll B(\log B)^{4}
$$

as required.

Acknowledgements. The main result in this paper was first established in the author's doctoral thesis, completed at the University of Oxford under the supervision of Roger Heath-Brown, and with the financial support of the Clarendon Fund, the National Sciences and Engineering Research Council of Canada, and Balliol College. 


\section{References}

[1] R. de la Bretèche and T. D. Browning, Sums of arithmetic functions over values of binary forms, Acta Arith. 125 (2006), 291-304.

[2] T. D. Browning, An overview of Manin's conjecture for del Pezzo surfaces, in: Analytic Number Theory, Clay Math. Proc. 7, Amer. Math. Soc., Providence, RI, 2007, $39-55$.

[3] T. D. Browning and D. R. Heath-Brown, Counting rational points on hypersurfaces, J. Reine Angew. Math. 584 (2005), 83-115.

[4] J. Franke, Yu. I. Manin and Yu. Tschinkel, Rational points of bounded height on Fano varieties, Invent. Math. 95 (1989), 421-435; erratum, ibid. 102 (1990), 463.

[5] Yu. I. Manin, Cubic Forms: Algebra, Geometry, Arithmetic, North-Holland, Amsterdam, 1974.

[6] T. Nagell, Introduction to Number Theory, 2nd ed., Chelsea, New York, 1964.

[7] M. Nair, Multiplicative functions of polynomial values in short intervals, Acta Arith. 62 (1992), 257-269.

[8] J. B. Rosser and L. Schoenfeld, Approximate formulas for some functions of prime numbers, Illinois J. Math. 6 (1962), 64-94.

Department of Pure Mathematics

Faculty of Mathematics

University of Waterloo

200 University Avenue West

Waterloo, ON, Canada N2L 3G1

E-mail: fsleung@math.uwaterloo.ca

Received on 10.6.2008

and in revised form on 16.8.2008 